\title{
Thymidylate synthase expression in primary colorectal cancer as a predictive marker for the response to 5-fluorouracil- and oxaliplatin-based preoperative chemotherapy for liver metastases
}

\author{
HIROSHI TAKEYAMA ${ }^{1}$, TOMOKO WAKASA ${ }^{2}$, KEISUKE INOUE ${ }^{1}$, KOTARO KITANI $^{1}$, MASANORI TSUJIE ${ }^{1}$, \\ TAKAFUMI OGAWA ${ }^{3}$, MASAO YUKAWA ${ }^{1}$, YOSHIO OHTA ${ }^{2}$ and MASATOSHI INOUE ${ }^{1}$ \\ Departments of ${ }^{1}$ Gastroenterological Surgery and ${ }^{2}$ Pathology, Kindai University Nara Hospital, Ikoma, \\ Nara 630-0293; ${ }^{3}$ Department of Chemical Science and Engineering, Graduate School of Engineering, \\ Kobe University, Kobe, Hyogo 657-8501, Japan
}

Received March 13, 2018; Accepted May 8, 2018

DOI: $10.3892 / \mathrm{mco} .2018 .1623$

\begin{abstract}
In patients with colorectal liver metastases (CRLM), predictive markers for response to preoperative chemotherapy are lacking. The aim of the present study was to evaluate the expression of thymidylate synthase (TS) and excision repair cross-complementation group 1 (ERCC1) as predictive markers in CRLM. A total of 24 patients with CRLM were included in this study. Tumor response was evaluated using the tumor regression grade (TRG) and Response Evaluation Criteria in Solid Tumors (RECIST) methods. TS and ERCC1 expression in paired CRLM and primary lesions were assessed by immunohistochemistry. We analyzed correlations between i) the response to preoperative chemotherapy evaluated by TRG and RECIST, ii) TS and ERCC1 expression and the response evaluated by TRG and RESICT, and iii) TS and ERCC1 expression in matched pairs of primary tumor and CRLM. The preoperative chemotherapy response evaluated by TRG and RECIST was significantly associated $(\mathrm{P}=0.0005)$. The response based on RECIST criteria and TRG was significantly associated with TS expression in the primary tumor $(\mathrm{P}=0.0272$, and $\mathrm{P}=0.0137$, respectively). No correlations were detected between marker expression in the primary tumor and in CRLM for either TS or ERCC1 ( $\mathrm{P}=0.371$ and $\mathrm{P}=1.00$, respectively). Our data suggested that TS expression in the primary tumor is a predictive marker of preoperative chemotherapy response in CRLM based on both TRG and RECIST methods.
\end{abstract}

Correspondence to: Dr Hiroshi Takeyama, Department of Gastroenterological Surgery, Kindai University Nara Hospital, Otoda-cho 1248-1, Ikoma, Nara 630-0293, Japan

E-mail: htakeyama.gesurg@gmail.com

Key words: colorectal cancer, colorectal liver metastases, preoperative chemotherapy, chemosensitivity, thymidylate synthase, excision repair cross-complementation group 1

\section{Introduction}

Surgical liver resection is the most effective treatment for colorectal liver metastases (CRLM) and is currently the only potentially curative therapeutic option $(1,2)$. Previously, preoperative chemotherapy was demonstrated to improve prognosis and increase conversion to resectability in patients with CRLM (3-5). However, preoperative chemotherapy is not always effective and the disease may progress (6). Therefore, it may be beneficial to personalize treatment based on the individual molecular characteristics of the tumor. Identification of predictive markers for the response to preoperative chemotherapy may help to ensure prompt selection of effective drugs for each patient and to avoid unnecessary administration of ineffective or even harmful drugs. Furthermore, the reduction in treatment costs would have economic benefits.

There are currently no molecular markers of chemosensitivity to predict the response of CRLM to 5-fluorouracil (5-FU)- and oxaliplatin-based treatment regimens, such as FOLFOX (5-FU, folinic acid and oxaliplatin) and XELOX (capecitabine and oxaliplatin). Thymidylate synthase (TS) and excision repair cross-complementation group 1 (ERCC1) have been shown to be useful predictors of the response to 5-FU- and oxaliplatin-based chemotherapy in colorectal cancer (CRC) (7-12). However, little is known on the direct association between TS and ERCC1 expression as detected by immunohistochemistry (IHC) of tumor tissues and the response to 5-FU- and oxaliplatin-based preoperative chemotherapy for CRLM. Furthermore, it is not known whether TS and ERCC1 expression levels in the primary lesions and CRLM are associated.

The aim of the present study was to evaluate TS and ERCC1 expression in primary lesions and CRLM as predictive markers for the response to preoperative chemotherapy according to both histological [tumor regression grade (TRG)] and radiological [Response Evaluation Criteria in Solid Tumors (RECIST)] assessments. Identification of predictive markers for response to chemotherapy may help identify the CRLM patients who would most benefit from preoperative chemotherapy. 


\section{Patients and methods}

Patients. The present study included 24 consecutive patients with CRLM who were treated with 5-FU- and oxaliplatin-based preoperative chemotherapy between January 2007 and February 2016. Selection of the chemotherapy regimen was not randomized, but rather determined by the clinician's preference. Medical records were reviewed and clinical data were retrospectively obtained. This study was conducted in compliance with the Declaration of Helsinki and in accordance with guidelines approved by the Institutional Research Board of Kindai University Nara Hospital (no. 364).

Immunohistochemistry. A total of 23 paired samples of formalin-fixed, paraffin-embedded sections from primary tumors and CRLM and 1 unpaired CRLM section were deparaffinized with xylene, rehydrated with a graded series of aqueous ethanol solutions, and then stained as briefly described herein. For antigen retrieval, the sections were placed in citrate buffer ( $\mathrm{pH}$ 6.0) and autoclaved at $121^{\circ} \mathrm{C}$ for $10 \mathrm{~min}$. Endogenous peroxidase activity was blocked by incubation of sections with a $3 \%$ hydrogen peroxide solution at room temperature for $15 \mathrm{~min}$, followed by rinsing with $0.05 \mathrm{M}$ phosphate-buffered saline (PBS) and blocking with Blocking One solution (Nacalai Tesque, Kyoto, Japan) at room temperature for $10 \mathrm{~min}$. The sections were then incubated with rabbit anti-TS monoclonal antibody (1:500, clone TS106, Dako, Tokyo, Japan) or mouse anti-ERCC1 monoclonal antibody (1:250, clone 8F1, Abcam, Cambridge, UK) overnight at $4^{\circ} \mathrm{C}$. Following washing with PBS, the sections were incubated with universal immuno-peroxidase polymer (N-Histofine Simple Stain MAX, Nichirei Co., Tokyo, Japan) at room temperature for $30 \mathrm{~min}$. After washing again with PBS, peroxidase activity was detected by incubation with 3,3'-diaminobenzidine tetrahydrochloride (DAB; Merck KGaA, Darmstadt, Germany) at room temperature for $5 \mathrm{~min}$. The sections were washed again with PBS, and cell nuclei were stained with Mayer's hematoxylin at room temperature for $1 \mathrm{~min}$. IHC qualitative scoring was performed using the ASCO/CAP criteria (13), i.e., 10\% of cells with nuclear staining was considered as a positive staining reaction for TS and ERCC1 (Fig. 1). The slides were examined independently by two pathologists blinded to the clinical data.

Imaging assessment. RECIST 1.1-based evaluation of the effect of preoperative chemotherapy was assessed by experienced gastroenterological surgeons using computed tomography or magnetic resonance imaging $(14,15)$. The criteria of the Japanese Society for Cancer of the Colon and Rectum (JSCCR) were used for grading liver metastasis. This JSCCR staging system is based on the number of liver metastases as follows: $\mathrm{H} 1, \leq 4$ metastatic tumors and the largest hepatic tumor sized $\leq 5 \mathrm{~cm} ; \mathrm{H} 2$, except $\mathrm{H} 1$ and $\mathrm{H} 3 ; \mathrm{H} 3, \geq 5$ metastatic tumors and the largest hepatic tumor sized $>5 \mathrm{~cm}$ (16).

Histological assessment. Two pathologists blinded to the patient's clinical information reviewed all histological specimens. Tumor and node staging for all resected specimens was
Table I. Patient characteristics $(n=24)$.

Characteristics No.

Age, years (median, range)

$64(39-78)$

Sex, male/female $16 / 8$

Tumor classification of the primary lesion

$\mathrm{T} 2 / \mathrm{T} 3 / \mathrm{T} 4$

Node classification

$\mathrm{N} 0 / \mathrm{N} 1 / \mathrm{N} 2 / \mathrm{N} 3$

$7 / 8 / 6 / 3$

Differentiation

Tub1/Tub2/muc

$7 / 16 / 1$

Location

Colon/rectum

Number of metastases

Multiple/solitary

Grade of liver metastases

$\mathrm{H} 1 / \mathrm{H} 2 / \mathrm{H} 3$

Size of largest metastasis prior to

chemotherapy, mm (median, range)

Size of largest metastasis after

chemotherapy, mm (median, range)

No. of chemotherapy cycles

(median, range)

Chemotherapy regimen

FOLFOX/XELOX

Molecular targeted drug

P-mab/C-mab/Bev

$8 / 1 / 11$

Response Evaluation Criteria in Solid

Tumors (RECIST)

$\mathrm{CR} / \mathrm{PR} / \mathrm{SD} / \mathrm{PD}$

$0 / 17 / 5 / 2$

Tumor Regression Grade (TRG)

$1 / 2 / 3 / 4 / 5$

$2 / 13 / 4 / 4 / 1$

ERCC1 expression in primary lesion

Negative/positive

ERCC1 expression in liver metastasis Negative/positive

$\mathrm{TS}$ expression in primary lesion

Negative/positive

TS expression in liver metastasis

Negative/positive

$18 / 6$

Tub1, well differentiated; tub2, moderately differentiated; muc, mucinous; FOLFOX, 5-fluorouracil, folinic acid and oxaliplatin; XELOX, capecitabine and oxaliplatin; P-mab, panitumumab; C-mab, cetuximab; Bev, bevacizumab; CR, complete response; PR, partial response; $\mathrm{SD}$, stable disease; $\mathrm{PD}$, progressive disease; $\mathrm{ERCC} 1$, excision repair cross-complementation group 1 ; TS, thymidylate synthase.

conducted according to the 7th American Joint Committee on Cancer TNM staging manual (17). The TRG method was used to characterize the tumor response (18) based on tumor viability and the extent of fibrosis and inflammation, and TRG classes 

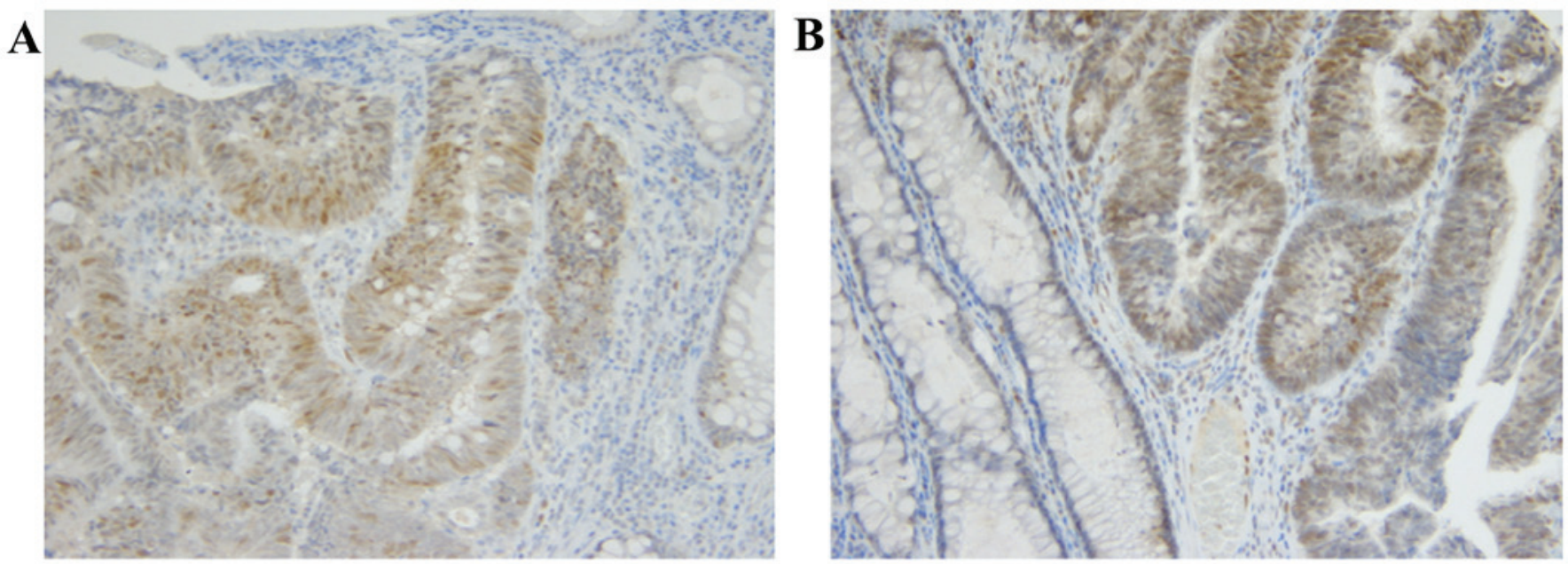

Figure 1. Representative images of immunohistochemical staining for TS and ERCC1 (original magnification, x200). (A) Positive TS expression; (B) Positive ERCC1 expression. TS, thymidylate synthase; ERCC1; excision repair cross-complementation group 1.

were defined as follows: TRG 1, complete regression with no residual tumor; TRG 2, presence of rare residual cancer cells; TRG 3, presence of larger numbers of residual cancer cells with predominant fibrosis; TRG 4, residual cancer outgrowing the fibrosis; and TRG 5, absence of regressive changes.

Statistical analysis. Statistical analysis was performed using JMP Pro ${ }^{\circledR}$ software, version 11 (SAS Institute, Cary, NC, USA). Continuous data are reported as median and range unless otherwise specified. Categorical data are presented as frequency and percentage. Comparison of continuous variables was performed using the Wilcoxon's rank-sum test. The Fisher's exact test was used for comparison of categorical variables as appropriate. Statistical significance was defined as $\mathrm{P}<0.05$.

\section{Results}

Patient characteristics. The patient characteristics are summarized in Table I. A total of 24 patients ( 8 women and 16 men) aged 39-78 years (median, 64 years) were analyzed in this study. A primary lesion specimen was missing for 1 patient who underwent surgery at another hospital. A total of 9 patients had rectal cancer and 15 patients had colon cancer. The chemotherapy regimens included modified FOLFOX6 (21 cases) and XELOX (3 cases). The median number of chemotherapy cycles was 6 (range, 3-56). Molecular-targeted drugs were administered to 20 patients: 8 patients received panitumumab, 1 received cetuximab, and 11 received bevacizumab. As regards the response to preoperative chemotherapy evaluated according to TRG, 2 patients were classified as TRG1, 13 as TRG2, 4 as TRG3, 4 as TRG4 and 1 as TRG5. As regards the response evaluated according to RECIST, 2 patients had progressive disease (PD), 5 had stable disease (SD), and 17 exhibited a partial response (PR). No patients achieved complete response (CR). Other clinical and histological data are provided in Table I.

Correlation between TRG and RECIST assessments. We analyzed the association between the response assessed
Table II. Correlation between response based on TRG and RECIST.

\begin{tabular}{lccc}
\hline & \multicolumn{2}{l}{ Response according to RECIST } & \\
\cline { 2 - 3 } TRG class & PR $(\mathrm{n}=17)$ & SD + PD (n=7) & P-value \\
\hline TRG1-3 $(\mathrm{n}=19)$ & 17 & 2 & 0.0005 \\
TRG4-5 $(\mathrm{n}=5)$ & 0 & 5 & \\
\hline
\end{tabular}

TRG, Tumor Regression Grade; RECIST, Response Evaluation Criteria In Solid Tumors; PD, progressive disease; PR, partial response; SD, stable disease; TRG1-3, responder; TRG4-5, non-responder.

by TRG and RECIST. Patients classified as TRG1-3 were considered to be responders, while those classified as TRG4-5 were considered to be non-responders (18-21). Based on this classification, 19 patients were assigned to the responder group (TRG 1-3) and 5 to the non-responder group (TRG 4-5). Similarly, patients were assigned to two groups based on RECIST criteria: responders (PR, $\mathrm{n}=17$ ) and non-responders (SD and PD, n=7) (22). The analysis identified a significant association between the response of CRLM patients to preoperative chemotherapy assessed by TRG and RECIST ( $\mathrm{P}=0.0005$; Table II).

Response to preoperative chemotherapy based on RECIST. The clinicopathological data for patients in the RECIST responder (PR) and non-responder (SD + PD) groups are summarized in Table III. The response based on RECIST was significantly associated with TS expression in the primary tumor and with the size of metastases prior to chemotherapy $(\mathrm{P}=0.0272$ and $\mathrm{P}=0.0454$, respectively). Other factors were not found to be significantly associated with RESICT.

Response to preoperative chemotherapy based on TRG. The clinicopathological data for patients in the TRG responder 
Table III. Response to preoperative chemotherapy based on RECIST.

\begin{tabular}{|c|c|c|c|}
\hline Variables & $\mathrm{PR}(\mathrm{n}=17)$ & $\mathrm{SD}+\mathrm{PD}(\mathrm{n}=7)$ & P-value \\
\hline Age, years (median, range) & $63(39-78)$ & $71(41-75)$ & 0.2151 \\
\hline \multicolumn{4}{|l|}{ Sex } \\
\hline Male & 13 & 3 & \multirow[t]{2}{*}{0.1670} \\
\hline Female & 4 & 4 & \\
\hline \multicolumn{4}{|l|}{ Tumor classification of the primary lesion } \\
\hline $\mathrm{T} 1, \mathrm{~T} 2$ & 1 & 0 & \multirow[t]{2}{*}{1.000} \\
\hline $\mathrm{T} 3, \mathrm{~T} 4$ & 16 & 7 & \\
\hline \multicolumn{4}{|l|}{ Node classification } \\
\hline No & 4 & 3 & \multirow[t]{2}{*}{0.3742} \\
\hline $\mathrm{N} 1, \mathrm{~N} 2$ & 13 & 4 & \\
\hline \multicolumn{4}{|l|}{ Location } \\
\hline Colon & 11 & 4 & \multirow[t]{2}{*}{1.000} \\
\hline Rectum & 6 & 3 & \\
\hline \multicolumn{4}{|l|}{ Number of metastases } \\
\hline Solitary & 2 & 3 & \multirow[t]{2}{*}{0.1265} \\
\hline Multiple & 15 & 4 & \\
\hline $\begin{array}{l}\text { Size of metastases prior to chemotherapy, } \mathrm{mm} \\
\text { (median, range) }\end{array}$ & $63.2(17.2-130.9)$ & $33.6(7.8-87.8)$ & 0.0454 \\
\hline \multicolumn{4}{|l|}{ Molecular targeted drug } \\
\hline P-mab, C-mab & 8 & 1 & \multirow[t]{3}{*}{0.0847} \\
\hline Bev & 8 & 3 & \\
\hline None & 1 & 3 & \\
\hline \multicolumn{4}{|l|}{ TS expression in primary lesion } \\
\hline Negative & 11 & 1 & \multirow[t]{2}{*}{0.0272} \\
\hline Positive & 5 & 6 & \\
\hline \multicolumn{4}{|l|}{ TS expression in liver metastasis } \\
\hline Negative & 13 & 5 & \multirow[t]{2}{*}{1.000} \\
\hline Positive & 4 & 2 & \\
\hline \multicolumn{4}{|l|}{ ERCC1 expression in primary lesion } \\
\hline Negative & 6 & 3 & \multirow[t]{2}{*}{1.000} \\
\hline Positive & 10 & 4 & \\
\hline \multicolumn{4}{|l|}{ ERCC1 expression in liver metastasis } \\
\hline Negative & 11 & 4 & \multirow[t]{2}{*}{1.000} \\
\hline Positive & 6 & 3 & \\
\hline
\end{tabular}

RECIST, Response Evaluation Criteria in Solid Tumors; tub1, well differentiated; tub2, moderately differentiated; muc, mucinous; FOLFOX, 5-flurouracil, folinic acid and oxaliplatin; XELOX, capecitabine and oxaliplatin; P-mab, panitumumab; C-mab, cetuximab; Bev, bevacizumab; PR, partial response; SD, stable disease; PD, progressive disease; ERCC1, excision repair cross-complementation group 1; TS, thymidylate synthase.

(TRG-3) and non-responder (TRG4-5) groups are summarized in Table IV. The response based on TRG was significantly associated with TS expression in the primary tumor $(\mathrm{P}=0.0137)$. Other factors were not found to be significantly associated with TRG class.

Correlation between TS and ERCCl expression in the primary lesion and CRCLM. As shown in Table V, no correlation was detected between TS expression in the primary lesion and that in the matched liver metastases $(\mathrm{P}=0.371)$.
There was also no correlation detected between ERCC1 expression in the primary lesion and that in the matched liver metastasis $(\mathrm{P}=1.00)$.

\section{Discussion}

The main methods used to assess the response of CRLM to chemotherapy include radiological and pathological grading systems (19,23). In radiological assessment, the effect of chemotherapy is usually evaluated on radiographic scans according to 
Table IV. Response to preoperative chemotherapy based on TRG.

\begin{tabular}{|c|c|c|c|}
\hline Variables & TRG1-3 (n=19) & TRG4-5 (n=5) & P-value \\
\hline Age, years (median, range) & $63(39-78)$ & $71(42-74)$ & 0.4339 \\
\hline \multicolumn{4}{|l|}{ Sex } \\
\hline Male & 14 & 2 & \multirow[t]{2}{*}{0.2885} \\
\hline Female & 5 & 3 & \\
\hline \multicolumn{4}{|l|}{ Tumor classification of the primary lesion } \\
\hline $\mathrm{T} 1, \mathrm{~T} 2$ & 1 & 0 & \multirow[t]{2}{*}{1.000} \\
\hline $\mathrm{T} 3, \mathrm{~T} 4$ & 18 & 5 & \\
\hline \multicolumn{4}{|l|}{ Node classification } \\
\hline N0 & 5 & 2 & \multirow[t]{2}{*}{0.6080} \\
\hline $\mathrm{N} 1, \mathrm{~N} 2$ & 14 & 3 & \\
\hline \multicolumn{4}{|l|}{ Location } \\
\hline Colon & 11 & 4 & \multirow[t]{2}{*}{0.6146} \\
\hline Rectum & 8 & 1 & \\
\hline \multicolumn{4}{|l|}{ Number of metastases } \\
\hline Solitary & 4 & 1 & \multirow[t]{2}{*}{1.000} \\
\hline Multiple & 15 & 4 & \\
\hline $\begin{array}{l}\text { Size of metastases before chemotherapy, mm } \\
\text { (median, range) }\end{array}$ & $56.2(17.2-30.9)$ & $18.4(7.8-87.8)$ & 0.0699 \\
\hline \multicolumn{4}{|l|}{ Molecular targeted drug } \\
\hline P-mab, C-mab & 8 & 1 & \multirow[t]{3}{*}{0.3385} \\
\hline Bev & 9 & 2 & \\
\hline None & 2 & 2 & \\
\hline \multicolumn{4}{|l|}{ TS expression in primary lesion } \\
\hline Negative & 12 & 0 & \multirow[t]{2}{*}{0.0137} \\
\hline Positive & 6 & 5 & \\
\hline \multicolumn{4}{|l|}{ TS expression in liver metastasis } \\
\hline Negative & 12 & 3 & \multirow[t]{2}{*}{1.000} \\
\hline Positive & 7 & 2 & \\
\hline \multicolumn{4}{|l|}{ ERCC 1 expression in primary lesion } \\
\hline Negative & 8 & 1 & \multirow[t]{2}{*}{0.6106} \\
\hline Positive & 10 & 4 & \\
\hline \multicolumn{4}{|l|}{ ERCC1 expression in liver metastasis } \\
\hline Negative & 14 & 4 & \multirow[t]{2}{*}{1.000} \\
\hline Positive & 5 & 1 & \\
\hline
\end{tabular}

TRG, Tumor Regression Grade; tub1, well differentiated; tub2, moderately differentiated; muc, mucinous; FOLFOX, 5-fluorouracil, folinic acid and oxaliplatin; XELOX, capecitabine and oxaliplatin; P-mab, panitumumab; C-mab, cetuximab; Bev, bevacizumab; PR, partial response; $\mathrm{SD}$, stable disease; $\mathrm{PD}$, progressive disease; ERCC1, excision repair cross-complementation group 1; TS, thymidylate synthase.

the RECIST scoring system. Recently, Rubbia et al published a novel grading system, TRG, which assesses prognosis based on the pathological response to chemotherapy (18). In the present study, we evaluated the expression of TS and ERCC1 in the primary colorectal lesion and CRLM to determine their potential as predictive markers of the response of CRLM to preoperative chemotherapy as assessed by both the TRG and RECIST methods.

The correlation between the TRG and RECIST results were first evaluated for the 24-patient cohort in the present study and observed a significant association between the two assessment tools. A previous study demonstrated that RECIST was significantly associated with the percentage of residual tumor cells in patients treated with preoperative chemotherapy for CRLM (24). In that study, the authors scored the pathological response semi-quantitatively (percentage of residual tumor cells relative to the total tumor surface area) and, although our study used a slightly different method of pathological assessment, our results are consistent with the findings of Chun et al, confirming that radiological assessment based on RECIST was significantly associated with the pathological assessment (24). 
Table V. Correlation between TS and ERCC1 expression in matched pairs of primary lesions and CRLM.

\begin{tabular}{lccc}
\hline & \multicolumn{2}{c}{$\begin{array}{c}\text { TS expression } \\
\text { in CRLM }\end{array}$} & \\
\cline { 2 - 3 } & Negative & Positive & P-value \\
\hline $\begin{array}{l}\text { TS expression in } \\
\text { primary lesion }\end{array}$ & & 0.371 \\
Negative & 10 & 2 & \\
Positive & 7 & 4 & \\
\hline
\end{tabular}

\begin{tabular}{lccc} 
& \multicolumn{2}{c}{$\begin{array}{c}\text { ERCC1 expression } \\
\text { in CRLM }\end{array}$} & \\
\cline { 2 - 3 } & Negative & Positive & P-value \\
\hline $\begin{array}{l}\text { ERCC1 expression } \\
\text { in primary lesion }\end{array}$ & & & 1.00 \\
Negative & 6 & 3 & \\
Positive & 8 & 6 & \\
\hline
\end{tabular}

TS, thymidylate synthase; ERCC1, excision repair cross-complementation group 1; CRLM, colorectal liver metastasis.

Fluoropyrimidines, particularly 5-FU, have been the mainstay of systemic treatment of metastatic CRC for $>50$ years. The major mechanism of action of 5-FU is inhibition of TS, which catalyzes a crucial rate-limiting step in DNA synthesis (25).

Several studies on metastatic CRC have demonstrated that high intratumoral TS levels are correlated with resistance to fluoropyrimidine treatment (26-28). Other studies have demonstrated that TS is a prognostic marker for patients with CRC (29) and metastatic CRC (30). Similar to the present study, Arienti et al demonstrated that TS expression is a marker of chemosensitivity of peritoneal carcinomatosis from colon cancer to 5-FU- and oxaliplatin-based chemotherapy (31). However, despite these promising results, TS has not been recommended for routine clinical practice as a predictor of response to 5-FU-based chemotherapy (32). Thus, the aim of the present study was to determine whether TS expression is a direct marker of the CRLM response to preoperative chemotherapy.

In this study, TS expression in the primary lesion, but not in CRLM, was identified as a predictive marker for the response to preoperative chemotherapy, as assessed by both TRG and RECIST. No significant difference in TS expression was found between the primary lesion andCRLM. Therefore, it appears that other molecular characteristics of the primary tumor must have been altered during the metastatic process (33). Chemotherapy may also have modified the tumor characteristics (34). We hypothesized that such factors may explain why TS expression in liver metastases was not a predictive marker of response.

ERCC1 expression was not found to be a predictive marker of response to preoperative chemotherapy. It was previously suggested that ERCC1 is a good predictive chemosensitivity marker for oxaliplatin-based chemotherapy (11); however, other studies have demonstrated that TS expression is a better predictive chemosensitivity marker compared with ERCC1 for 5-FU- and oxaliplatin-based chemotherapy $(28,31,35,36)$. In agreement with the latter reports, we found that TS expression is a more useful predictor of chemosensitivity to 5-FU- and oxaliplatin-based chemotherapy compared with ERCC1 expression. Although ERCC1 was a good predictive marker for oxaliplatin-based chemotherapy, previous reports included several factors, such as inclusion criteria, outcome and stage, and it remains controversial whether it is also a direct good predictive marker for response to 5-FU- and oxaliplatin-based chemotherapy.

There were certain limitations to the present study. First, this was a small, retrospective, non-randomized study, and the results may have been affected by its retrospective design. The inclusion or exclusion criteria for preoperative chemotherapy were not strictly defined. We included only patients who proceeded to receive surgery, whereas patients with CRLM who failed to convert to resectability were excluded. Thus, the patients with the lowest responses may have been excluded from this study.

Ideally, these problems could be overcome by performing liver biopsies before and after chemotherapy. However, liver biopsy is not practically recommended due to the risk of tumor spillage, which may be the cause of peritoneal carcinomatosis (37). For this reason, only specimens resected by surgery were evaluated. Second, we did not analyze prognosis in terms of disease-free survival and overall survival, mainly because prognosis was significantly affected by the postoperative treatment. Therefore, we considered that the chemotherapy response based on pathological and radiological assessments would allow for direct analysis of the association between protein expression and the tumor response to therapy.

In summary, the results of the present study demonstrated a significant association between TS expression in the primary colorectal tumor and response to preoperative chemotherapy as assessed by both TRG and RECIST. Although investigations of larger patient cohorts are required to confirm our results, the data of the present study suggest that TS expression in the primary lesion may be a predictive marker for the response of CRLM to 5-FU- and oxaliplatin-based preoperative chemotherapy.

\section{Acknowledgements}

Not applicable.

\section{Funding}

No funding was received.

\section{Availability of data and materials}

All data generated or analyzed during this study are included in this published article.

\section{Authors' contributions}

HT designed the study and analyzed the data. HT, KK, KI, MT, MY and MI performed the surgery. TW, TO, and YO 
performed the histological experiments. HT, MY, and MI wrote the manuscript.

\section{Ethics approval and consent to participate}

Appropriate ethical approval was obtained from the Institutional Review Board of Kindai University Nara Hospital (Nara, Japan; no. 364). Patient consent was not required for the present study, as it was conducted retrospectively.

\section{Consent for publication}

Not applicable.

\section{Competing interests}

The authors confirm that they have no competing interests.

\section{References}

1. Rees M, Tekkis PP, Welsh FK, O'Rourke T and John TG: Evaluation of long-term survival after hepatic resection for metastatic colorectal cancer: A multifactorial model of 929 patients. Ann Surg 247: 125-135, 2008.

2. van der Pool AE, de Wilt JH, Lalmahomed ZS, Eggermont AM, Ijzermans JN and Verhoef C: Optimizing the outcome of surgery in patients with rectal cancer and synchronous liver metastases. Br J Surg 97: 383-390, 2010.

3. Goéré D, Deshaies I, de Baere T, Boige V, Malka D, Dumont F, Dromain C, Ducreux M and Elias D: Prolonged survival of initially unresectable hepatic colorectal cancer patients treated with hepatic arterial infusion of oxaliplatin followed by radical surgery of metastases. Ann Surg 251: 686-691, 2010.

4. Giacchetti S, Itzhaki M, Gruia G, Adam R, Zidani R, Kunstlinger F, Brienza S, Alafaci E, Bertheault-Cvitkvoic F, Jasmin C, et al: Long-term survival of patients with unresectable colorectal cancer liver metastases following infusional chemotherapy with 5-fluorouracil, leucovorin, oxaliplatin and surgery. Ann Oncol 10: 663-669, 1999.

5. Tanaka K, Adam R, Shimada H, Azoulay D, Lévi F and Bismuth H: Role of neoadjuvant chemotherapy in the treatment of multiple colorectal metastases to the liver. Br J Surg 90: 963-969, 2003.

6. Adam R, Pascal G, Castaing D, Azoulay D, Delvart V, Paule B, Levi $\mathrm{F}$ and Bismuth $\mathrm{H}$ : Tumor progression while on chemotherapy: A contraindication to liver resection for multiple colorectal metastases? Ann Surg 240: 1052-1064. 2004

7. Johnston PG, Lenz HJ, Leichman CG, Danenberg KD, Allegra CJ, Danenberg PV and Leichman L: Thymidylate synthase gene and protein expression correlate and are associated with response to 5 -fluorouracil in human colorectal and gastric tumors. Cancer Res 55: 1407-1412, 1995.

8. Li S, Zhu L, Yao L, Xia L and Pan L: Association between ERCC1 and TS mRNA levels and disease free survival in colorectal cancer patients receiving oxaliplatin and fluorouracil (5-FU) adjuvant chemotherapy. BMC Gastroenterol 14: 154, 2014.

9. Choueiri MB, Shen JP, Gross AM, Huang JK, Ideker T and Fanta P: ERCC1 and TS expression as prognostic and predictive biomarkers in metastatic colon cancer. PLoS One 10: e0126898, 2015.

10. Noda $E$, Maeda $K$, Inoue $T$, Fukunaga $S$, Nagahara $H$, Shibutani M, Amano R, Nakata B, Tanaka H, Muguruma K, et al: Predictive value of expression of ERCC 1 and GST-p for 5-fluorouracil/oxaliplatin chemotherapy in advanced colorectal cancer. Hepatogastroenterology 59: 130-133, 2012.

11. Geva R, Shamai S, Brazowsky E, Paoulas M, Ben-Haim M, Johnstone E, Alex B and Shacham-Shmueli E: The predictive role of ERCC1 status in oxaliplatin based neoadjuvant therapy for metastatic colorectal cancer (mCRC) to the liver. Cancer Invest 33: 89-97, 2015.

12. Han JJ, Baek SK, Lee JJ, Kim GY, Kim SY and Lee SH: Combination of TRAP1 and ERCC1 expression predicts clinical outcomes in metastatic colorectal cancer treated with Oxaliplatin/5-fluorouracil. Cancer Res Treat 46: 55-64, 2014.
13. Wolff AC, Hammond ME, Hicks DG, Dowsett M, McShane LM, Allison KH, Allred DC, Bartlett JM, Bilous M, Fitzgibbons P, et al: Recommendations for human epidermal growth factor receptor 2 testing in breast cancer: American society of clinical oncology/college of american pathologists clinical practice guideline update. J Clin Oncol 31: 3997-4013, 2013.

14. Trillet-Lenoir V, Freyer G, Kaemmerlen P, Fond A, Pellet O, Lombard-Bohas C, Gaudin JL, Lledo G, Mackiewicz R, Gouttebel MC, et al: Assessment of tumour response to chemotherapy for metastatic colorectal cancer: Accuracy of the RECIST criteria. Br J Radiol 75: 903-908, 2002.

15. Eisenhauer EA, Therasse P, Bogaerts J, Schwartz LH, Sargent D, Ford R, Dancey J, Arbuck S, Gwyther S, Mooney M, et al: New response evaluation criteria in solid tumours: revised RECIST guideline (version 1.1). Eur J Cancer 45: 228-247, 2009.

16. Japanese Society for Cancer of the Colon and Rectum. General Rules for Clinical and Pathological Studies on Cancer of the Colon, Rectum and Anus, 7th edition. Tokyo: Japanese Society for Cancer of the Colon and Rectum, 2006.

17. Edge SB, Byrd DR, Compton CC, Fritz AG, Greene FL and Trotti A, (eds): AJCC cancer staging manual 7th edition. Springer NY, New York, 2010.

18. Rubbia-Brandt L, Giostra E, Brezault C, Roth AD, Andres A, Audard V, Sartoretti P, Dousset B, Manjo PE, Soubrane O, et al: Importance of histological tumor response assessment in predicting the outcome in patients with colorectal liver metastases treated with neo-adjuvant chemotherapy followed by liver surgery. Ann Oncol 18: 299-304, 2007.

19. Dede K, Salamon F, Landherr L, Jakab F and Bursics A: Pathologic assessment of response to chemotherapy in colorectal cancer liver metastases after hepatic resection: which method to use? Pathol Oncol Res 21: 173-179, 2015.

20. Noble F, Hopkins J, Curtis N, Kelly JJ, Bailey IS, Byrne JP, Bateman AC, Bateman AR and Underwood TJ: The role of systemic inflammatory and nutritional blood-borne markers in predicting response to neoadjuvant chemotherapy and survival in oesophagogastric cancer. Med Oncol 30: 596, 2013.

21. Fareed KR, Al-Attar A, Soomro IN, Kaye PV, Patel J, Lobo DN, Parsons SL and Madhusudan S: Tumour regression and ERCC1 nuclear protein expression predict clinical outcome in patients with gastro-oesophageal cancer treated with neoadjuvant chemotherapy. Br J Cancer 102: 1600-1607, 2010.

22. Valtorta E, Misale S, Sartore-Bianchi A, Nagtegaal ID, Paraf F, Lauricella C, Dimartino V, Hobor S, Jacobs B, Ercolani C, et al: KRAS gene amplification in colorectal cancer and impact on response to EGFR-targeted therapy. Int J Cancer 133: 1259-1265, 2013.

23. Nishioka Y, Shindoh J, Yoshioka R, Gonoi W, Abe H, Okura N, Yoshida S, Oba M, Hashimoto M, Watanabe G, et al: Radiological morphology of colorectal liver metastases after preoperative chemotherapy predicts tumor viability and postoperative outcomes. J Gastrointest Surg 19: 1653-1661, 2015.

24. Chun YS, Vauthey JN, Boonsirikamchai P, Maru DM, Kopetz S, Palavecino M, Curley SA, Abdalla EK, Kaur H, Charnsangavej C and Loyer EM: Association of computed tomography morphologic criteria with pathologic response and survival in patients treated with bevacizumab for colorectal liver metastases. JAMA 302: 2338-2344, 2009.

25. Van Triest B, Pinedo HM, Giaccone G and Peters GJ: Downstream molecular determinants of response to 5-fluorouracil and antifolate thymidylate synthase inhibitors. Ann Oncol 11: 385-391, 2000.

26. Bertino JR and Banerjee D: Is the measurement of thymidylate synthase to determine suitability for treatment with 5-fluoropyrimidines ready for prime time? Clin Cancer Res 9: 1235-1239, 2003.

27. Popat S, Matakidou A and Houlston RS: Thymidylate synthase expression and prognosis in colorectal cancer: A systematic review and meta-analysis. J Clin Oncol 22: 529-536, 2004.

28. Leichman CG, Lenz HJ, Leichman L, Danenberg K, Baranda J, Groshen S, Boswell W, Metzger R, Tan M and Danenberg PV: Quantitation of intratumoral thymidylate synthase expression predicts for disseminated colorectal cancer response and resistance to protracted-infusion fluorouracil and weekly leucovorin. J Clin Oncol 15: 3223-3229, 1997.

29. Shirota Y, Stoehlmacher J, Brabender J, Xiong YP, Uetake H, Danenberg KD, Groshen S, Tsao-Wei DD, Danenberg PV and Lenz HJ: ERCC1 and thymidylate synthase mRNA levels predict survival for colorectal cancer patients receiving combination oxaliplatin and fluorouracil chemotherapy. J Clin Oncol 19: 4298-4304, 2001. 
30. Silvestris N, Simone G, Partipilo G, Scarpi E, Lorusso V, Brunetti AE, Maiello E, Paradiso A and Mangia A: CES2, ABCG2, TS and Topo-I primary and synchronous metastasis expression and clinical outcome in metastatic colorectal cancer patients treated with first-line FOLFIRI regimen. Int J Mol Sci 15: 15767-15777, 2014.

31. Arienti C, Tesei A, Verdecchia GM, Framarini M, Virzi S, Grassi A, Scarpi E, Turci L, Silverstrini R, Amadori D and Zoli W: Role of conventional chemosensitivity test and tissue biomarker expression in predicting response to treatment of peritoneal carcinomatosis from colon cancer. Clin Colorectal Cancer 12: 122-127, 2013.

32. Locker GY, Hamilton S, Harris J, Jessup JM, Kemeny N, Macdonald JS, Somerfield MR, Hayes DF and Bast RC Jr; ASCO: ASCO 2006 update of recommendations for the use of tumor markers in gastrointestinal cancer. J Clin Oncol 24: 5313-5327, 2006.

33. Lim B, Mun J, Kim JH, Kim CW, Roh SA, Cho DH, Kim YS and Kim JC: Genome-wide mutation profiles of colorectal tumors and associated liver metastases at the exome and transcriptome levels. Oncotarget 6: 22179-22190, 2015.

34. Baba H, Baba Y, Uemoto S, Yoshida K, Saiura A, Watanabe M, Maehara Y, Oki E, Ikeda Y, Matsuda H, et al: Changes in expression levels of ERCC1, DPYD, and VEGFA mRNA after first-line chemotherapy of metastatic colorectal cancer: Results of a multicenter study. Oncotarget 6: 34004-34013, 2015.
35. López-Gómez M, Moreno-Rubio J, Suárez-García I, Cejas P, Madero R, Casado E, Jiménez A, Sereno M, Gómez-Raposo C, Zambrana F, et al: SMAD4 and TS expression might predict the risk of recurrence after resection of colorectal liver metastases. Clin Trans Oncol 17: 133-138, 2015.

36. Kumamoto K, Kuwabara K, Tajima Y, Amano K, Hatano S, Ohsawa T, Okada N, Ishibashi K, Haga N and Ishida H: Thymidylate synthase and thymidine phosphorylase mRNA expression in primary lesions using laser capture microdissection is useful for prediction of the efficacy of FOLFOX treatment in colorectal cancer patients with liver metastasis. Oncol Lett 3: 983-989, 2012

37. Jones OM, Rees M, John TG, Bygrave S and Plant G: Biopsy of resectable colorectal liver metastases causes tumour dissemination and adversely affects survival after liver resection. $\mathrm{Br} \mathrm{J}$ Surg 92: 1165-1168, 2005.

This work is licensed under a Creative Commons Attribution-NonCommercial-NoDerivatives 4.0 International (CC BY-NC-ND 4.0) License. 